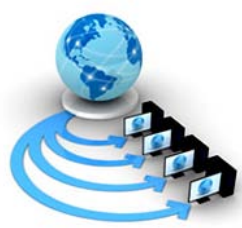

Volume 9, No. 3, May-June 2018

International Journal of Advanced Research in Computer Science

RESEARCH PAPER

Available Online at www.ijarcs.info

\title{
THE STUDY OF MEDICAL IMAGE RETRIEVAL SYSTEM USING CBIR
}

\author{
Fatema Jamnagarwala \\ Computer Engineering \\ Sipna College Of Engineering and Technology \\ Amravati.India
}

\author{
Dr.V.K Shandilya \\ Computer Engineering \\ Sipna College of Engineering and Technology \\ Amravati,India
}

\begin{abstract}
Image retrieval systems search through the info to list Associate in Nursing array of pictures in keeping with the question unemployed..They notice the photographs that ar terribly kind of like the question unemployed. This work has been developed to aim Associate in Nursing economical visual-Content-based technique to look, browse and retrieve relevant pictures from large-scale of medical image collections throughout the image retrieval, options play the important role. There are numerous options that are extracted options like texture, color, intensity, shape, resolution, international and native options etc. Our main eye is on the precise medical domain wherever it's abundant required.Color is such a feature which will not influence be a awfully helpful technique as a result of the medical systems typically need to upset the grey scale mode pictures. The options explored ar intensity, texture. initial it extracts the feel feature and therefore the intensity feature from the given input image. The ensuring image is compared to the array of pictures within the information the highest most resembling pictures are then retrieved from the information.
\end{abstract}

Keywords: medical image, digital image, retrieval of images, indexes

\section{INTRODUCTION}

Content based image retrieval is an image retrieval technique based on automatic matching of the features of the image query the digital image searching downside for giant databases that creates use of the features of the photographs instead of fully relying on matter info given by users like captions and key-words. For terribly giant databases or mechanically generated (security systems, drugs etc.) images, in person describing and adding notes to each image is long. instead of betting on manual classification and text description for each image, low-level visual options mechanically extracted ar used for illustration of the image content. Content-based image retrieval makes use of the visual contents of a picture to represent and index the image. Color, shape, texture, and spacial layout are few generally used contents.

The "content-based image retrieval" was fictional in 1992 because it was employed by T. Kato to elaborate experiments into automatic retrieval of pictures from a info, betting on the colours and shapes. the foremost earliest business CBIR system was developed by IBM and was called QBIC (Query by Image Content).[1][2][6]

\section{CONTENT BASED IMAGE RETRIEVAL:OVERVIEW}

All Content-based image retrieval (CBIR)system involves at least four steps which leads us to successful image retrieval :

1. First step includes feature extraction and indexing of image according to visual features such as shape, texture and color.

2. Next step extracts features of query image fired.In other words feature extraction of query image takes place.

3. Now, the system matches query image to the most resembling and similar images in the database according to some image similarity protocol. Thus this steps forms the search part of Content-based image retrieval (CBIR).

4. The display of the outcomesare evaluated by user interface and feedback, and these are arranged according the type of the user-interaction by rectifying the search through some automatic or manual preference scheme,etc.[5]

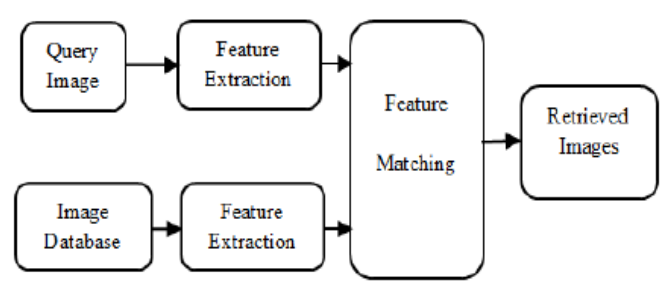

Figure1 Generic CBIR System [14]

\section{FEATURE EXTRACTION AND INDEXING}

Feature extraction is a standout amongst the most imperative advance in picture order. It is utilized to extricate the component of a picture as superbly as could be expected under the circumstances. These systems are utilized to get the component that will be helpful in grouping and understanding the pictures.

There are different element extraction procedures. The techniques are delegated low-level component extraction and High-level element extraction. [3]

Low-level element extractions depend on finding the focuses, lines, edge, and so on while abnormal state include extraction techniques utilize the low level element to give more noteworthy data to additionally handling of picture examination. Highlight extraction manages the different properties of pictures, for example, shading, shape and surface etc.[3][13] 


\section{A.Color}

Shading speaks to a standout amongst the most generally utilized visual highlights in CBIR systems.A shading space is utilized to speak to shading pictures. Regularly, RGB (red Green Blue) space, where the dark level power is spoken to as the aggregate of red, green and blue dim level forces, is honed broadly .The quantity of containers of the histogram decides the shading quantization.[12]

\section{B. Shapes}

Shape extraction, which incorporates edges, forms, turns into a set up field in itself in picture preparing and different strategies are currently imagined. Various highlights normal for objects shape, which are generally not reliant on size or introduction, are ascertained for each protest of picture database,for picture recovery. Thus, some separated highlights are utilized to coordinate two pictures recovered. Inquiries are then replied by processing a similar arrangement of highlights for the question picture, and recovering those put away pictures whose highlights most intently match of the inquiry. [12]

\section{C.Texture}

Surface is one more vital property of pictures. It alludes to the visual examples that have properties of likenesses or game plan that don't come about because of the nearness of just a solitary shading or force. Different surface portrayals have been explored in design acknowledgment and PC vision. An assortment of techniques has been utilized for surface similitude; the best-settled strategy depends totally on looking at estimations of what are known as second-arrange measurements figured from question and put away images.[12]

\section{QUERY TECHNIQUES USED IN CBIR}

CBIR method is been utilized as a part of a large number of the frameworks by utilizing inquiry by illustrations which needs a fitting picture for questioning. Client can plan and change questions on the authorization of intuitive recovery interface .It gives a case of yield as wanted and requesting that the framework recover assist cases of a similar kind.

\section{A. Semantic retrieval}

Semantic recovery manages a solicitations made by client like discover pictures of Steve Jobs such open finished undertaking is extremely intense for PCs to perform. Along these lines numerous substance based picture frameworks by and large make utilization of lower-level highlights like surface, shading, and shape. These highlights are either utilized as a part of mix with interfaces. In any case, picture recovery requires human input with a specific end goal to distinguish more elevated amount features.[4][14]

\section{B. Relevance Feedback(Human interaction)}

Mix of CBIR look methods out there with the wide determination of clients and their motivation are frequently an intense to be returned from the offered question to assemble clients criticism to utilize the information we tend to retrieved.[4][14]

\section{Iterative/MachineLearning}

Machine learning is usually connected with and joins up with computational insights, that furthermore points its spotlights on forecast making through the PCs utilization. it's a very strong cling to numerical improvement, that conveys methodologies, hypothesis and application spaces to the circle. These strategies have turned out to be very basic in CBIR.[4][14]

\section{Content Compearison using image data measure}

This is the most well-known technique utilized for contrasting two pictures in CBIR framework is utilizing a picture remove measure. This separations are looked at by the closeness of two pictures in different measurements, for example, shading, surface, shape and others. For instance a correct counterpart for inquiry means correct separation zero.[4][14]

\section{APPLiCATION OF CBIR IN MEDICAL IMAGE RETRIEVAL SYSTEM}

In this paper we have considered the applying and capability of CBIR in therapeutic picture recovery framework. The snappy and expanding assortment of advanced picture stockpiling frameworks in clinical routine, for example, $\mathrm{X}$ beam (CT), reverberation (MR),magnetic reverberation spectrometry (MRS), single check boson discharge pc imaging (SPECT), antielectron outflow imaging (PET), ultrasound, electrical source(ESI), electrical resistivity imaging (EIT), rises requests for fresh out of the plastic new procedures to utilize. Content-based picture recovery has been anticipated by the wellbeing calling for involving into picture chronicling and correspondence frameworks (PACS).[1]

To every one of the radiologists, doctors, pros, facilities, and imaging focus design of PACS coordinated imaging modalities and interfaces with clinic and division information frameworks to deal with its stockpiling and appropriation of pictures.

In this paper we tend to propose Associate in Nursing medicinal picture store and recovery procedure upheld totally unique separated alternatives like picture visual diagram investigation, extraction of shading esteems from mesmeric picture Associate in Nursing coherent shape discovery of a therapeutic picture. along these lines we'll specified new procedure which will encourage medicinal proclamation is content-based picture retrieval).[1][6]

\section{VI . MEDICAL CONTENT BASED IMAgE RETRIEVAL METHODS}

There are 2 routes that inside which the therapeutic pictures are recovered, content based generally and substance based procedures. starting method is recovery pictures by content based recovered. amid this method, physically message depictions are given and old information strategy oversaw pictures are utilized. in spite of the fact that content based systems are very fast and dependable once pictures are elegantly composed, they can't seek in unannotated picture databases.

The content based picture recovery has the ensuing downsides, since it needs time extreme comment strategies. In contentbased restorative picture recovery method, pictures in information arrangement by visual substance like shading, shape and surface and so forth. CBIR frameworks are created uncommon systems.[5]

\section{A .Image Enhancement}

A picture improvement can be utilized better from visual substance then for each picture in the picture database from where they are extricated. The way toward enhancing the 
nature of a carefully put away picture is prepared by controlling the picture with programming. It is very simple, for instance, to make a picture lighter or darker, or to increment or reduction differentiate. So the improvement of pictures are improved the situation the advancement of the substance. Consequently picture upgrade assumes an essential part in therapeutic recovery system.[5] [7][8]

\section{B.Feature Extraction}

A CBIR framework has two essential segments. The principal part picture is highlights/descriptors. This segment speaks to the visual data and goes for filling the hole between the visual substance and its numerical speak to.The second part is likenesses examination, which accommodates scope of similitudes between picture highlights in light of scientific analysis.[5][7][8]

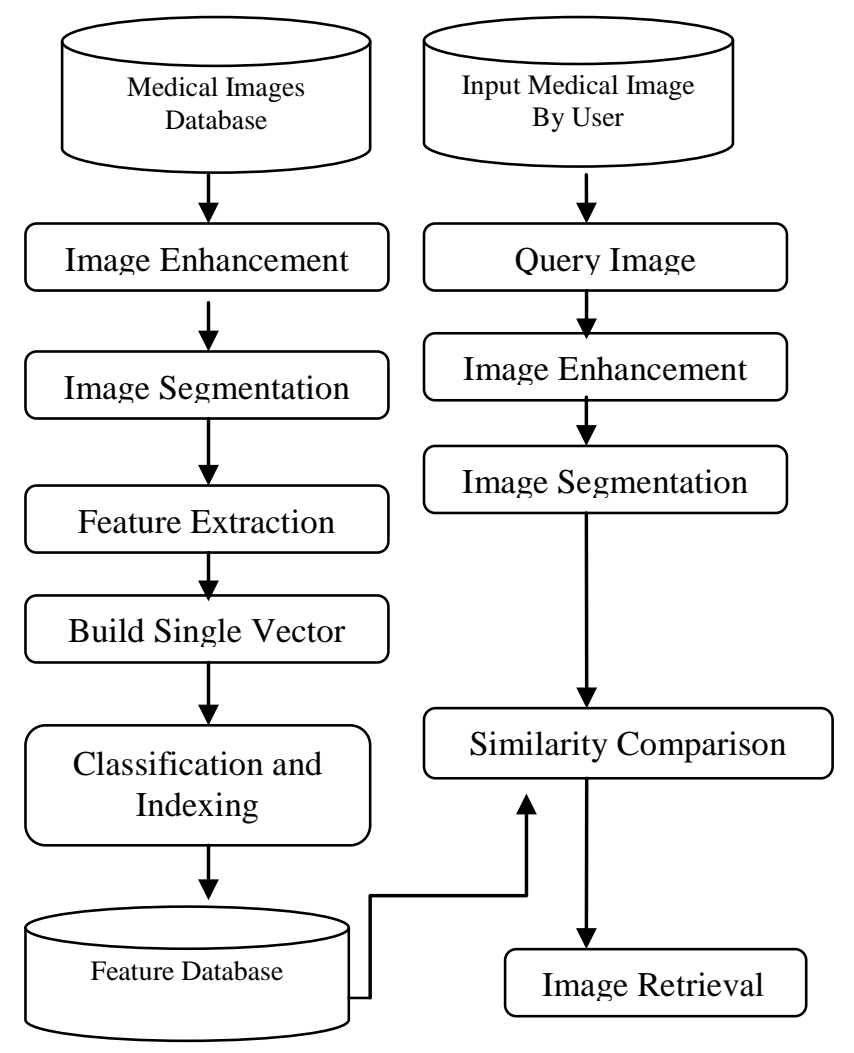

Figure 2. Architecture of CBIR systems in medical[5]

\section{ImageSegmentation}

The point of image segementation is to bunch pixels into fundamental picture parts, i.e. The division is to modify and adjust the delineation of a photo into one thing that is a great deal of pregnant and simpler to check and dissect. Picture division systems in medicinal are regularly arranged into 2 wide classes area based and shape based for the most part approaches. Area based procedures are a great deal of ready to diagram an overall goal work. Shape based methodologies regularly start with an essential phase of edge identification, trailed by a connecting technique that tries to exploit continuity.[5][7][8]

\section{EXISTING CONTENT BASED IMAGE RETRIEVAL SYSTEMS IN MEDICAL}

- Although content-based image retrieval has frequently been proposed for use in therapeutic and restorative picture administration, just a couple of substance based recovery frameworks have been produced particularly for medicinal pictures.

- Only a couple of substance based recovery frameworks have been produced particularly for therapeutic pictures like ASSERT(Automatic Search and Selection Engine with Retrieval Tools),CasImage, IRMA(Image Retrieval in Medical Applications),NHANESII.

\section{A. ASSERT}

- ASSERT (Automated Search and decision Engine with Retrieval Tools) a CBIR framework is utilized for the space of HRCT (High Resolution Computed Tomography) photos of the respiratory organ with emphysema-type maladies.

- $\quad$ Purdue University, Indiana University, and University of Wisconsin Hospital, USA created Automatic Search and Selection Engine with Retrieval Tools.

- It is a CBIR framework for the space of HRCT (Highdetermination Computed Tomography) photos of the respiratory organ with emphysema-type sicknesses.

- It removes 255 alternatives of surface, shape, edges, and dim scale properties in pathology-bearing regions..[1] [6]

B. CasImage

- CasImage is another therapeutic CBIR framework. College Hospital of Geneva, European nation built up this restorative CBIR framework alluded to as CasImage..

- $\quad$ The picture information contains kind of pictures from CT, MRI, and radiographs, to paint photographs. this technique, it contains an educating and reference information, and accordingly the recovery framework, that is modified from the ASCII content document GIFT (GNU Image Finding Tool).

- This recovery framework isolates global and territorial shading and surface alternatives, that includes 166 hues inside the shading region alluded to as HSV. along these lines various combos of issue names and visual choices are utilized for therapeutic recovery of images.[1][6]

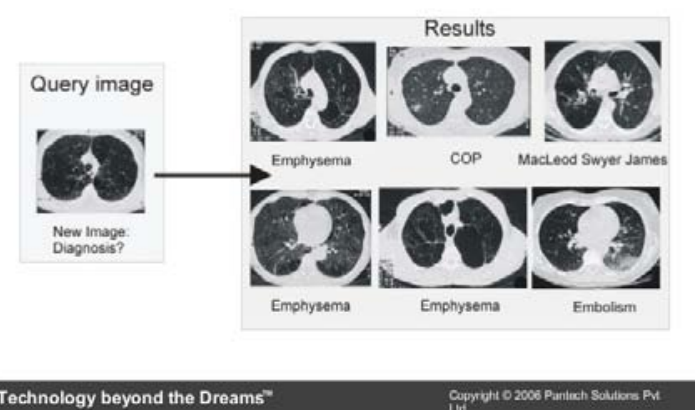

Figure3. Medical Image Retrieval Example [14]

\section{IRMA (Image Retrieval in Medical Applications)}


- IRMA (Image Retrieval in Medical Applications) framework built and created by urban focus University of Technology, Deutschland abuse various imaging modalities and procedures.

- The IRMA framework is thusly utilized as a stage and base for content-based picture recovery in medicinal applications and frameworks

- This framework hence parts the picture recovery technique into seven stages together with classification, enrollment, include extraction ,highlight decision, ordering, recognizable proof, andretrieval.[1][6]

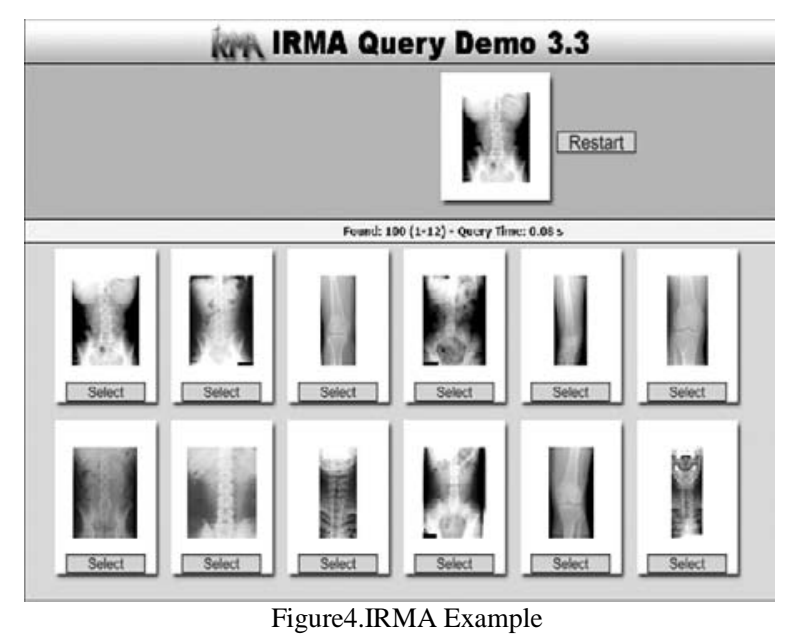

\section{OTHER APPLICAIONS OF CBIR}

A wide exhibit of conceivable applications for CBIR innovation has been distinguished. Without a doubt productive regions include:

- Crime counteractive action

- The military

- Intellectual property Architectural and building outline

- $\quad$ Fingerprint Identification

- Journalism and promoting

- Biodiversity Information frameworks

- Geographical data and remote detecting frameworks

- Cultural legacy

- Digital Library

- Home excitement

- Web searching[10]

\section{CONCLUSION}

Content-based info retrieval in little question encompasses a giant potential within the medical domain. These contentbased strategies are often used on an oversized variety and sort of pictures and in an exceedingly wide space of applications out there. Thus, it is clear that new tools and strategies ar required to manage the increasing and growing quantity of visual info that's created in medical establishments and systems.For the betterment of the CBIR system performance in numerous completely different hardware platforms, having completely different technical capabilities and conditions, CBIR systems typically examines image content via the lowlevel options (color, texture and shape). Improved CBIR applications involves rising the retrieval performance, efficiency, ability and measurability of the system. therefore at the top all we will say is , the content based mostly access strategies have a larger potential once utilized in the incoming ways that.

content based mostly medical image retrieval schemes noted during this paper ar promising still there's way more scope for future analysis. this analysis work improves the understanding of varied techniques for feature extraction and similarity mensuration that connects medical image retrieval and advances the state-of the art through its contributions.[11]

\section{REFERENCES}

[1] Ivica Dimitrovski, Dejan Gorgevik, Suzana Loskovska "WebBased Medical Image Retrieval System"Faculty of Electrical Engineering and Information Technology.

[2] R. Datta, D. Joshi, J. Li, J.Z. Wang,” Image Retrieval: Ideas, Influences, and Trends of the New Age, ACM Transactions on Computing Surveys”, Vol. No 20.

[3] Rajkumar Goel, Vineet Kumar, Saurabh Srivastava,A. K. Sinha” A Review of Feature Extraction Techniques for Image Analysis” Vol. 6, Special Issue 2, February 2017, IJARCCE.

[4] https://en.wikipedia.org/wiki/Contentbased_image_retrieval

[5] Y. Fanid Fathabad ,M.A. Balafar,” CONTENT BASED IMAGE RETRIEVAL FOR MEDICAL IMAGES”Vol.4,Issue 12,September2012,Pages177-182.

[6] E. Acar, S. Arslan, A. Yazici, M. Koyuncu, Slim-Tree and Bit matrix Index Structures in Image Retrieval System Using MPEG-7 Descriptors, Sixth International Workshop on Content-Based Multimedia Indexing (CBMI-2008), 2008.

[7] https://link.springer.com/chapter/10.1007/978-3-64232112-2_16

[8] Gurmeet Kaur and Er. Arshdeep Singh “A Review Paper on Content Based Image Retrieval” Kaur et al., International Journal of Advanced Research in Computer Science and Software Engineering 5(4), pp. 1404-1406, April- 2015,

[9] Tobias Weyand, Thomas Deselaers, Combining Contentbased Image Retrieval with Textual Information Retrieval, RWTH Aachen October 2005

[10] http://www.enggjournals.com/ijcse/doc/IJCSE11-03-04030.pdf

[11] Chi-Ren Shyu, ASSERT: A Physician-in-the-loop Content-Based Retrieval System for HRCT Image Databases, Computer Vision and Image Understanding: CVIUASSERT, 1999

[12] http://www.gnu.org/software/gift/

[13] http://www.irmaproject

[14] org/projekte_en.php?SELECTED=00085.

[15] http://en.wikipedia.org/ 